\title{
A CHARACTERISTIC PROPERTY OF THE SPACE $s$
}

\author{
DIETMAR VOGT
}

(Communicated by Thomas Schlumprecht)

\begin{abstract}
It is shown that under certain stability conditions a complemented subspace of the space $s$ of rapidly decreasing sequences is isomorphic to $s$ and this condition characterizes $s$. This result is used to show that, for the classical Cantor set $X$, the space $C_{\infty}(X)$ of restrictions to $X$ of $C^{\infty}$-functions on $\mathbb{R}$ is isomorphic to $s$, which leads to an improvement of the theory developed in a previous work of the author.
\end{abstract}

\section{INTRODUCTION}

In the present note we study the space $s$ of rapidly decreasing sequences, that is, the space

$$
s=\left\{x=\left(x_{0}, x_{1}, \ldots\right): \lim _{n} x_{n} n^{k}=0 \text { for all } k \in \mathbb{N}\right\} .
$$

Equipped with the norms $\|x\|_{k}=\sup _{n}\left|x_{n}\right|(n+1)^{k}$ it is a nuclear Fréchet space. It is isomorphic to many of the Fréchet spaces which occur in analysis, in particular, spaces of $C^{\infty}$-functions.

It is easily seen that instead of the sup-norms we might use the norms

$$
|x|_{k}=\left(\sum_{n}\left|x_{n}\right|^{2}(n+1)^{2 k}\right)^{1 / 2}
$$

which makes $s$ a Fréchet-Hilbert space.

More generally, we define for any sequence $\alpha: 0 \leq \alpha_{0} \leq \alpha_{1} \leq \nearrow+\infty$ the power series space of infinite type

$$
\Lambda_{\infty}(\alpha):=\left\{x=\left(x_{0}, x_{1}, \ldots\right):|x|_{t}^{2}=\sum_{n=0}^{\infty}\left|x_{n}\right|^{2} e^{2 t \alpha_{n}}<\infty \text { for all } t>0\right\} .
$$

Equipped with the hilbertian norms $|\cdot|_{k}, k \in \mathbb{N}_{0}$, it is a Fréchet-Hilbert space. It is nuclear if and only if $\lim \sup _{n} \log n / \alpha_{n}<\infty$. With this definition $s=\Lambda_{\infty}(\alpha)$ with $\alpha_{n}=\log (n+1)$.

A Fréchet space with the fundamental system of seminorms $\|\cdot\|_{0} \leq\|\cdot\|_{1} \leq \ldots$ has property $(\mathrm{DN})$ if

$$
\exists p \forall k \exists K, C>0:\|\cdot\|_{k}^{2} \leq C\|\cdot\|_{p}\|\cdot\|_{K} \cdot
$$

In this case $\|\cdot\|_{p}$ is called a dominating norm.

$E$ has property $(\Omega)$ if

$$
\forall p \exists q \forall m \exists 0<\theta<1, C>0:\|\cdot\|_{q}^{*} \leq C\|\cdot\|_{p}^{* \theta}\|\cdot\|_{m}^{* 1-\theta} .
$$

Received by the editors May 16, 2013 and, in revised form, July 10, 2013. 2010 Mathematics Subject Classification. Primary 46A45, 46A63, 46E10.

Key words and phrases. Space $s$, stability condition, Cantor set. 
Here we set for any continuous seminorm $\|\cdot\|$ and $y \in E^{\prime}$ the dual, extended real valued, norm $\|y\|^{*}=\sup \{|y(x)|: x \in E,\|x\| \leq 1\}$.

By Vogt-Wagner [10] a Fréchet space $E$ is isomorphic to a complemented subspace of $s$ if and only if it is nuclear and has properties (DN) and $(\Omega)$.

It is a longstanding unsolved problem of the structure theory of nuclear Fréchet spaces, going back to Mityagin, whether every complemented subspace of $s$ has a basis. If it has a basis, then it is isomorphic to some power series space $\Lambda_{\infty}(\alpha)$. The space $\Lambda_{\infty}(\alpha)$ to which it is isomorphic, if it has a basis, can be calculated in advance by a method going back to Terzioğlu [6] which we describe now.

Let $X$ be a vector space and $A \subset B$ absolutely convex subsets of $X$. We set

$\delta_{n}(A, B):=\inf \{\delta>0$ : exists linear subspace $F \subset X, \operatorname{dim} F \leq n$ with $A \subset \delta B+F\}$.

It is called the $n$-th Kolmogoroff diameter of $A$ with respect to $B$.

If now $E$ is a complemented subspace of $s$, that is, $E$ is nuclear and has properties $(\mathrm{DN})$ and $(\Omega)$, then we choose $p$ such that $\|\cdot\|_{p}$ is a dominating norm and for $p$ we choose $q>p$ according to property $(\Omega)$. We set

$$
\alpha_{n}=-\log \delta_{n}\left(U_{q}, U_{p}\right)
$$

where $U_{k}=\left\{x \in E:\|x\|_{k} \leq 1\right\}$. The space $\Lambda_{\infty}(\alpha)$ is called the associated power series space and $E \cong \Lambda_{\infty}(\alpha)$ if it has a basis.

If $\lim \sup _{n} \alpha_{2 n} / \alpha_{n}<\infty$, then, by Aytuna-Krone-Terzioğlu [2, Theorem 2.2], $E \cong \Lambda_{\infty}(\alpha)$. This is, in particular, the case if $E$ is stable, that is, if $E \oplus E \cong E$.

For all that and further results of structure theory of infinite type power series spaces see [8]. For results and unexplained notation of general functional analysis see 5 .

\section{MAin RESUlt}

Lemma 2.1. Let $E$ be a complemented subspace of $s,\|\cdot\|_{0}$ a dominating hilbertian norm and $\|\cdot\|_{1}$ a hilbertian norm chosen for $\|\cdot\|_{0}$ according to $(\Omega)$. If there is a linear isomorphism $\psi: E \oplus E \rightarrow E$ such that

$$
\begin{aligned}
\|x\|_{0}+\|y\|_{0} & \leq C_{0}\|\psi(x \oplus y)\|_{0}, \\
\|\psi(x \oplus y)\|_{1} & \leq C_{1}\left(\|x\|_{1}+\|y\|_{1}\right),
\end{aligned}
$$

then $E \cong s$.

Proof. For $x \oplus y \in E \oplus E$ we set $\|(x, y)\|_{0}:=\left(\|x\|_{0}^{2}+\|y\|_{0}^{2}\right)^{1 / 2}$ and $\|(x, y)\| \|_{1}:=$ $\left(\|x\|_{1}^{2}+\|y\|_{1}^{2}\right)^{1 / 2}$. With new constants $C_{k}$ we have

$$
\|\| x \oplus y \mid\left\|_{0} \leq C_{0}\right\| \psi(x \oplus y) \|_{0} \text { and }\|\psi(x \oplus y)\|_{1} \leq C_{1}\|\| x \oplus y \mid \|_{1} .
$$

To calculate the associated power series space for $E$ we set:

$$
\begin{aligned}
& \alpha_{n}=-\log \delta_{n}\left(U_{1}, U_{0}\right) \text { where } U_{k}=\left\{x \in E:\|x\|_{k} \leq 1\right\}, \\
& \beta_{n}=-\log \delta_{n}\left(V_{1}, V_{0}\right) \text { where } V_{k}=\left\{x \oplus y \in E \oplus E:\|x \oplus y\|_{k} \leq 1\right\} .
\end{aligned}
$$

Due to the estimates (2.1) we have

$$
\frac{1}{C_{1}} \psi\left(V_{1}\right) \subset U_{1} \subset U_{0} \subset C_{0} \psi\left(V_{0}\right)
$$

and therefore

$$
\delta_{n}\left(V_{1}, V_{0}\right)=\delta_{n}\left(\psi V_{1}, \psi V_{0}\right) \leq C_{0} C_{1} \delta_{n}\left(U_{1}, U_{0}\right)
$$


which implies

with $d=\log C_{0} C_{1}$.

$$
\alpha_{n} \leq \beta_{n}+d
$$

By explicit calculation of the Schmidt expansion of the canonical map $j_{1}^{0}$ between the local Hilbert spaces of $\||\cdot|\|_{1}$ and $\left|\|\cdot \mid\|_{0}\right.$ and by use of the fact that singular numbers and Kolmogoroff diameters coincide, we obtain that $\beta_{2 n}=\beta_{2 n+1}=\alpha_{n}$ for all $n \in \mathbb{N}_{0}$.

Therefore we have $\alpha_{2 n} \leq \beta_{2 n}+d=\alpha_{n}+d$ for all $n \in \mathbb{N}_{0}$ and this implies $\alpha_{2^{k}} \leq \alpha_{1}+k d$ for all $k \in \mathbb{N}_{0}$. For $n \in \mathbb{N}$ we find $k \in \mathbb{N}$ such that $2^{k-1} \leq n \leq 2^{k}$ and we obtain $\alpha_{n} \leq \alpha_{2^{k}} \leq \alpha_{1}+k d \leq\left(\alpha_{1}+d\right)+d \log n$.

Since $E \subset s$, which implies the left inequality below, we have shown that there is a constant $D>0$ such that

$$
\frac{1}{D} \log n \leq \alpha_{n} \leq D \log n
$$

for large $n \in \mathbb{N}$. This implies that $\Lambda_{\infty}(\alpha)=s$.

A Fréchet-Hilbert space $E$ is called normwise stable if it admits a fundamental system of hilbertian seminorms for which there is an isomorphism $\psi: E \oplus E \rightarrow E$ such that

$$
\frac{1}{C_{k}}\left(\|x\|_{k}+\|y\|_{k}\right) \leq\|\psi(x \oplus y)\|_{k} \leq C_{k}\left(\|x\|_{k}+\|y\|_{k}\right)
$$

for all $k$. Since, clearly, $s$ is normwise stable we have shown.

Theorem 2.2. $E \cong s$ if and only if $E$ is isomorphic to a complemented subspace of $s$ and normwise stable.

We may also express Lemma 2.1 in the following way:

Theorem 2.3. Let the Fréchet-Hilbert space $E$ be a complemented subspace of $s$, $\|\cdot\|_{0}$ a dominating norm and $\|\cdot\|_{1}$ be a norm chosen according to $(\Omega)$. Let $P$ be a linear projection in $E$, continuous with respect to $\|\cdot\|_{0}$. We set $E_{1}=R(P)$, $E_{2}=N(P)$ and assume that there are linear isomorphisms $\psi_{j}: E \rightarrow E_{j}, j=1,2$, continuous with respect to $\|\cdot\|_{1}$ such that $\psi^{-1}$ is continuous with respect to $\|\cdot\|_{0}$. Then $E \cong s$.

Proof. We set $\psi(x \oplus y):=\psi_{1}(x)+\psi_{2}(y)$ and obtain with suitable constants:

$$
\begin{aligned}
& \|x\|_{0}+\|y\|_{0} \leq C^{\prime}\left(\left\|\psi_{1}(x)\right\|_{0}+\left\|\psi_{2}(y)\right\|_{0}\right) \leq C_{0}\left\|\psi_{1}(x)+\psi_{2}(y)\right\|_{0}=C_{0}\|\psi(x \oplus y)\|_{0} \\
& \|\psi(x \oplus y)\|_{1}=\left\|\psi_{1}(x)+\psi_{2}(y)\right\|_{1} \leq\left\|\psi_{1}(x)\right\|_{1}+\left\|\psi_{2}(y)\right\|_{1} \leq C_{0}\left(\|x\|_{1}+\|y\|_{1}\right) .
\end{aligned}
$$

Lemma 2.1 yields the result.

\section{Applichtion}

An interesting application of this result is the following. Let $X \subset[0,1]$ be the classical Cantor set and $C_{\infty}(X):=\left\{\left.f\right|_{X}: f \in C^{\infty}[0,1]\right\}=\left\{\left.f\right|_{E}: f \in C^{\infty}(\mathbb{R})\right\}$. The space $C_{\infty}(X)$ equipped with the quotient topology is a nuclear Fréchet space and, since $C^{\infty}[0,1] \cong s$ isomorphic to a quotient of $s$, hence has property $(\Omega)$. By a theorem of Tidten [7] it has also property (DN). Therefore it is isomorphic to a complemented subspace of $s$ (see [10]).

We should remark that, due to the fact that $X$ is perfect, we have $C_{\infty}(X)=\mathcal{E}(X)$ where $\mathcal{E}(X)$ denotes the space of Whitney jets on $X$, for which Tidten's result is formulated. 
By obvious identifications we have

$$
C_{\infty}(X) \cong C_{\infty}(X \cap[0,1 / 3]) \oplus C_{\infty}(X \cap[2 / 3,1]) \cong C_{\infty}(X) \oplus C_{\infty}(X)
$$

and it is easily seen that this establishes normwise stability. Therefore we have shown

Theorem 3.1. If $X$ is the classical Cantor set, then $C_{\infty}(X) \cong s$.

It should be remarked that spaces of Whitney jets on Cantor-type sets have been under investigation by several authors. Theorem 3.1 could also be derived by a combination of results in the literature. In Arslan-Goncharov-Kocatepe 1 it has been shown that for the Cantor set $X$ the diametral dimensions of $\mathcal{E}(X)$ and $s$ coincide, from where, by means of the Aytuna-Krone-Terzioğlu Theorem or also using a result in Goncharov 4, one can derive the result. Another way would be by the combination of results in Bialas-Volberg [3] and Zériahi [1]. Still our proof has the advantage of giving a quick, direct and purely functional-analytic access. It can also be generalized since the (DN)-property is well investigated and known for wider classes of Cantor-type sets (see e.g. [1,7]).

Finally we want to indicate another interesting consequence of our result. By $A^{\infty}$ we denote the space of all $2 \pi$ periodic functions on $\mathbb{R}$ for which all negative Fourier coefficients vanish and by $A_{\infty}(X)$ the space of restrictions of $A^{\infty}$-functions to $X$, both equipped with their natural topologies. In [9, Proposition 5.1] it was shown that $A_{\infty}(X)=C_{\infty}(X)$ and this was used to show that $A_{\infty}(X)$ has a basis (see [9, Theorem 5.3]). By use of Theorem 3.1 this can be improved as follows:

Theorem 3.2. If $X$ is the classical Cantor set, then $A_{\infty}(X) \cong s$.

\section{ACKNOWLEDGMENTS}

The author wants to thank T. Ciaś and the referee for useful remarks, bringing to his attention the relation of Theorem 3.1 to the existing literature.

\section{REFERENCES}

[1] Bora Arslan, Alexander P. Goncharov, and Mefharet Kocatepe, Spaces of Whitney functions on Cantor-type sets, Canad. J. Math. 54 (2002), no. 2, 225-238, DOI 10.4153/CJM-2002007-3. MR1892995 (2003a:46041)

[2] A. Aytuna, J. Krone, and T. Terzioğlu, Complemented infinite type power series subspaces of nuclear Fréchet spaces, Math. Ann. 283 (1989), no. 2, 193-202, DOI 10.1007/BF01446430. MR.980593 (90a:46014)

[3] Leokadia Białas and Alexander Volberg, Markov's property of the Cantor ternary set, Studia Math. 104 (1993), no. 3, 259-268. MR.1220665(94h:41024)

[4] A. Goncharov, Bases in the spaces of $C^{\infty}$-functions on Cantor-type sets, Constr. Approx. 23 (2006), no. 3, 351-360, DOI 10.1007/s00365-005-0598-5. MR2201471 (2006k:46036)

[5] Reinhold Meise and Dietmar Vogt, Introduction to functional analysis, Oxford Graduate Texts in Mathematics, vol. 2, The Clarendon Press Oxford University Press, New York, 1997. Translated from the German by M. S. Ramanujan and revised by the authors. MR,1483073 (98g:46001)

[6] T. Terzioğlu, On the diametral dimension of some classes of F-spaces (English, with Turkish summary), J. Karadeniz Univ. Fac. Arts Sci. Ser. Math.-Phys. 8 (1985), 1-13. MR.924467 (88j:46006)

[7] Michael Tidten, Kriterien für die Existenz von Ausdehnungsoperatoren zu $\mathfrak{E}(K)$ für kompakte Teilmengen $K$ von $\mathbf{R}$ (German), Arch. Math. (Basel) 40 (1983), no. 1, 73-81, DOI 10.1007/BF01192754. MR720896 (85g:46043) 
[8] D. Vogt, Structure theory of power series spaces of infinite type (English, with English and Spanish summaries), RACSAM. Rev. R. Acad. Cienc. Exactas Fís. Nat. Ser. A Mat. 97 (2003), no. 2, 339-363. MR2068185 (2005e:46011)

[9] Dietmar Vogt, Restriction spaces of $A^{\infty}$, Rev. Mat. Iberoam. 30 (2014), no. 1, 65-78, DOI 10.4171/RMI/769. MR3186931

[10] Dietmar Vogt and Max Josef Wagner, Charakterisierung der Quotientenräume von $s$ und eine Vermutung von Martineau (German, with English summary), Studia Math. 67 (1980), no. 3, 225-240. MR592388 (81k:46002)

[11] Ahmed Zériahi, Inegalités de Markov et développement en série de polynômes orthogonaux des fonctions $C^{\infty}$ et $A^{\infty}$ (French), Several complex variables (Stockholm, 1987/1988), Math. Notes, vol. 38, Princeton Univ. Press, Princeton, NJ, 1993, pp. 683-701. MR.1207888 (94a:41018)

FB Math.-Nat., Bergische Universität Wuppertal, Gauss-Str. 20, 42119 Wuppertal, GERMANY

E-mail address: dvogt@math.uni-wuppertal.de 